\title{
An alternative approach to the analysis of mixed cropping experiments. 1. Estimation of competition effects
}

\section{J. T. Spitters}

Department of Theoretical Production Ecology, Agricultural University, P.O. Box 430, 6700 AK Wageningen, Netherlands

Received 5 April 1982; accepted 24 August 1982

Key-words: mixed cropping, competition, model, maize, groundnut.

\section{Summary}

A simple model is introduced to estimate the degree of intra-specific competition, inter-specific competition and niche differentiation from final biomass data of a set of populations varying in species composition and total density. To estimate these competition effects, the model does not require replacement series but can deal with addition series. The model is illustrated with results of mixed cropping of maize and groundnut.

\section{Introduction}

Especially in the tropics, research on mixed cropping receives much attention ( $\mathrm{Pa}$ pendick et al., 1976). In those studies, mixtures of different species composition are grown besides monocrops at different densities. Often, the mixture that yields most in the particular experiment is concluded to be the optimal one, without analysing the effects in more detail. A more thorough analysis would improve understanding of the effects of mixed cropping and would facilitate effective search for high-yielding mixtures. However, mixture effects are difficult to interpret and the few, quantitative analyses are not very satisfactory (review by Willey, 1979).

In this paper an approach will be presented that brings together a number of newly developed ideas with existing procedures. It meets practical demands more than existing methods of analysis. In this paper (Part 1) competition effects are estimated on the basis of biomass data, while in a subsequent paper (Part 2, Spitters 1983 ) the effects of mixture composition and density on marketable yield are analysed.

\section{Experimental data}

The approach is illustrated with the results of experiments with maize and groundnut. The data were kindly provided by W. C. H. van Hoof (Dept. of Tropical Crop 


\section{J. T. SPITTERS}

Science, Agricultural University, Wageningen) and collected during a co-operation project between the Agricultural University, Wageningen and the Brawijaya University, Malang, East Java, Indonesia. The comprehensive results of his research on mixed cropping of groundnut and maize will be published soon.

In the experiments the maize cv. Kretek and the groundnut cv. Gajah were used. The maize variety has a growth period of about 83 days, while that of the groundnut is about 108 days. Most data are derived from an experiment carried out in 1980 where replacement series (de Wit, 1960) were grown at different total densities. Within each series, one maize plant was replaced by two groundnut plants. Maize was sown 9 days after groundnut. The populations were grown in plots of $20 \mathrm{~m}^{2}$ in a randomized complete block design with four replications.

Figs. 1 and 2 (Part 1) are based on an experiment in 1977 where a fan design (Nelder, 1962) is used. Two fans of maize were established: one fan involved a monocrop giving a range of maize densities in monoculture, the other was laid out in a farmers' field of groundnut where the groundnuts were grown at a recommended density of 16 plants $\mathrm{m}^{-2}$. Maize was sown 31 days after groundnut. Four replicates were laid out with both fans being present in each replicate. Fig. 2 of Part 2 (Spitters, 1983) is based on a similar fan experiment from 1980, but with three replicates and maize sown 10 days after groundnut.

\section{A simple model to quantify competition effects}

\section{Biomass as criterion}

Plants compete with each other for the available growth factors like light, water, nitrogen and minerals. Those resources are distributed among the plants, with each plant trying to fulfil its needs. Biomass production is approximately linear to the uptake of that resource that limits growth, so that the distribution of the limiting resource among the plants reflects itself in their biomasses. Interplant competition is better measured by biomass than by the yield of any plant part, because dry-matter distribution within the plant varies with the competitive stress.

\section{Intra-specific competition (density response)}

The most simple way of interplant competition is that between plants of the same species. This intra-specific competition expresses itself in the response of biomass to plant density (Fig. 1a).

Several equations have been used to describe this density response (Willey \& Heath, 1969). For reasons wich will be clarified later, we take the hyperbola:

$$
y=N /\left(b_{0}+b_{1} N\right)
$$

where $y$ is the biomass in $\mathrm{g} \mathrm{m}^{-2}, N$ is the plant density in numbers $\mathrm{m}^{-2}$ and $b_{0}$ and $b_{1}$ are constants.

From this equation the average weight per plant is derived as

$$
w=y / N=1 /\left(b_{0}+b_{1} N\right)
$$



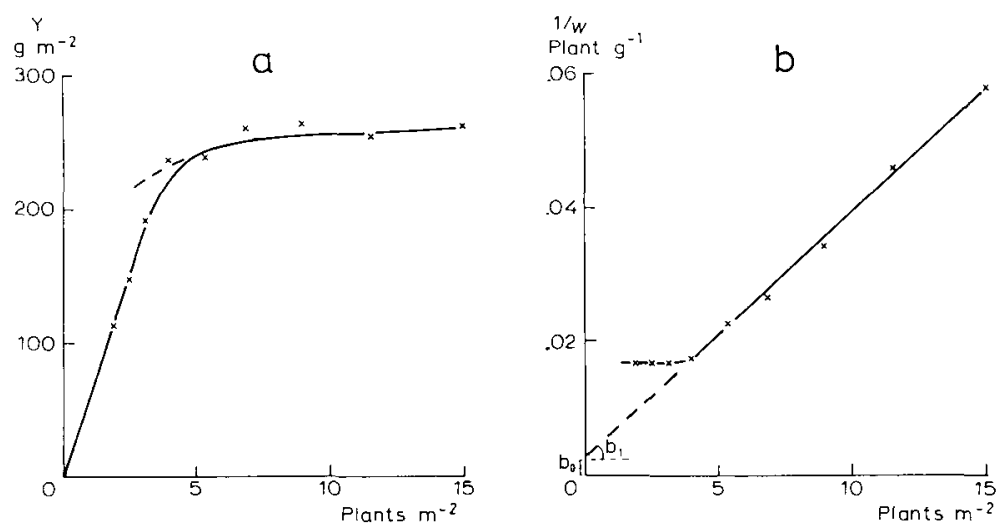

Fig. 1. Density response of maize. Plots of (a) biomass per unit area and (b) the reciprocal of per-plant biomass against plant density.

with $w$ in g plant $^{-1}$. To estimate $b_{0}$ and $b_{1}$ this expression is written in the linear regression form

$$
1 / w=b_{0}+b_{1} N
$$

where $b_{0}$ is the intercept and $b_{1}$ is the slope (Fig. 1b). Because of the heterogeneity of variances around the line a weighted regression analysis (Appendix 1) should be used.

The intercept $b_{0}$ is the reciprocal of the virtual biomass of an isolated plant. At very wide spacings there is no interplant competition so that the per-plant weight remains constant with decreasing density and does not increase as is suggested by the hyperbolic equation. The observed biomass of an isolated plant is therefore somewhat smaller than the apparent biomass measured by $1 / b_{0}$ (Fig. $1 \mathrm{~b}$ ).

The slope $b_{1}$ measures how $1 / w$ increases, and hence how the per-plant weight $w$ decreases with any plant added to the population. The ratio $b_{1} / b_{0}$ expresses this increase of $1 / w$ relative to its value without competition so that it may be used as a measure of intra-specific competitive stress. The coefficient $b_{1}$ is the reciprocal of the maximum biomass per unit area achieved at infinite density. This can be seen by taking the limit

$$
\operatorname{Lim}_{N \rightarrow \infty} \frac{1}{y}=\operatorname{Lim}_{N \rightarrow \infty} \frac{1}{w N}=\operatorname{Lim}_{N \rightarrow \infty}\left(\frac{b_{0}}{N}+b_{1}\right)=b_{1}
$$

\section{Inter-specific competition (mixture effect)}

Not only plants of the same species but also plants of different species compete with each other when they are growing in the same field. If adding plants of the own species affects $1 / w$ additively, then it seems reasonable to assume that adding plants of another species affects $1 / w$ also additively. We can then write the reciprocal of the 
per-plant weight of species 1 in mixture with species 2 in the multiple linear regression form

$$
1 / w_{1}=b_{1,0}+b_{1,1} N_{1}+b_{1,2} N_{2}
$$

The first subscript of the regression coefficients denotes the species which biomass is considered, while the second subscript points to the associated species. To estimate the regression coefficients and their standard errors, a weighted analysis is preferable (Appendix 1). The equation can easily be expanded to cover mixtures of more than two species.

We saw already that $b_{1}$ measures the effect of intra-specific competition. The coefficient $b_{2}$ quantifies the effect of inter-specific competition. In this way intra- and inter-specific competition are separated mathematically.

From the regression equation we see that adding one plant of species 1 has an equal effect on $1 / w_{1}$, as adding $b_{1,1} / b_{1,2}$ plants of species 2 . We might say that species 1 is a $b_{1,1} / b_{1.2}$ times as strong a competitor for itself as species 2 is for species 1 . This is displayed graphically in Fig. 2. These data meet the assumption of additivity: the influence of the fixed number of groundnut plants on $1 / w$ of maize remains equal of size, independent of the number of maize plants (parallel lines).

\section{Assumptions}

It is assumed that:

- The density response of the biomass of a species can be described with a rectangular hyperbola. Many experiments show that this is a reasonable assumption (e.g. de Wit, 1960, pp. 31-44).

- Inter-specific competition can be incorporated in the hyperbolic equation in a way similar to intra-specific competition. More precisely, the effect of the number of plants of a species $i$ on its reciprocal per-plant weight $1 / w_{i}$ is additive to that of the number of plants of another species $j$ on $1 / w_{i}$. The results of Fig. 2 fit in with this assumption and from Appendix 2 it can be shown that the results of de Wit (1960, pp. 63-67) also support this assumption.

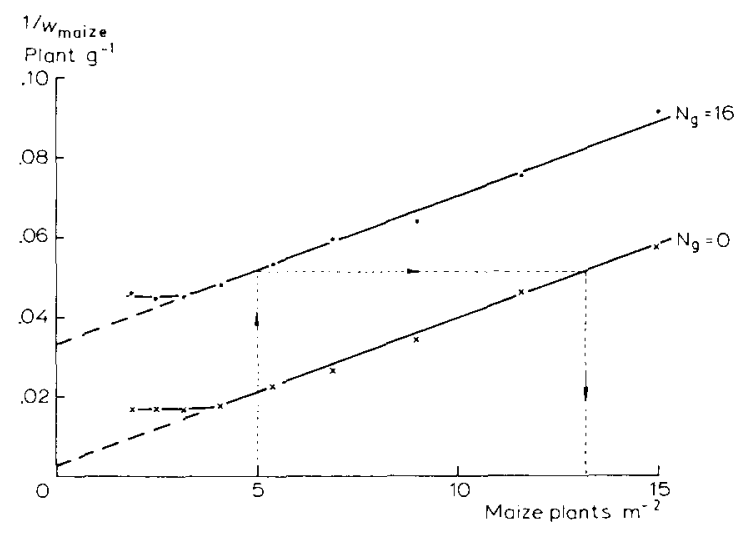

Fig. 2. Addition of 16 groundnut plants $\left(N_{g}=16\right)$ to a monocrop of maize $\left(N_{g}=\right.$ $0)$ has the same effect on $1 / w$ of maize as adding 8 maize plants. Thus, for a maize plant the presence of 2 groundnut plants is similar to the presence of one other maize plant. Because of the parallel lines, this substitution holds independent of the relative frequencies of the species and independent of total population density. 
- This additivity implies that the competition effects, measured by the regression coefficients, are independent of plant numbers, i.e. independent of the mutual frequencies of the species in the population as well as of total population density. The generally observed frequency independence of the relative crowding coefficient in replacement series with a fixed total population density (de Wit, 1960; Braakhekke, 1980) denotes that the assumption of frequency independence of competition effects at a given total population density is justified for most experiments. The relation between the competition parameters used here and the relative crowding coefficient of de Wit (1960) is explained in Appendix 2. The results of Fig. 2 fit in with independence of the regression coefficients from total density. However, more experimental evidence is needed to confirm that independence of total density.

- The model does not account for the effects of the spatial arrangement of the plants on the competitive relations. Hence, it is assumed that the spatial arrangement is either the same for alle populations or has no influence. The latter holds when interplant competition is of a diffuse nature. Diffuse competition will be approximated better in mixed cropping with species randomly distributed in the field or mixed within rows than in intercropping with species grown in separate rows (Spitters, 1979, p. 46).

- The field where the plants grow is homogeneous.

\section{Backgrounds of the model and relation with other models}

The equations are based on the description of the biomass-density response with a rectangular hyperbola. The advantages of the rectangular hyperbola are:

- the parameters and their standard errors can be estimated in a simple way as regression coefficients and their standard errors, after transforming the equation to a multiple linear regression form;

- interpretation of the effect of varying the number of the same species (intra-specific competition) as well as that of the other species (inter-specific competition) is simple because plant numbers are in a linear, additive form in the equation.

Extension of the linearized hyperbolic equation with an interaction term $b_{3} N_{1} N_{2}$, to allow for deviations from additivity, would complicate the interpretation and improves results only slightly.

Other functions may be used, but they do not combine the advantages of the rectangular hyperbola. For example, also the equation

$$
y_{1}=b_{1.0}+b_{1,1} \ln N_{1}+b_{1,2} \ln N_{2}
$$

for biomass $y_{1}$ per unit area showed a good fit, but additivity of $\ln N$, i.e. multiplicity of $N$, is less easy to interpret.

Several authors have developed models to account for the effects of intra- and inter-specific competition (reviews by Trenbath, 1978; Spitters, 1979, Chapter 3). Most of these models may be transposed into each other to some degree. The approach presented in this paper is related to that given by de Wit (1960); the differences and similarities being treated in Appendix 2. 


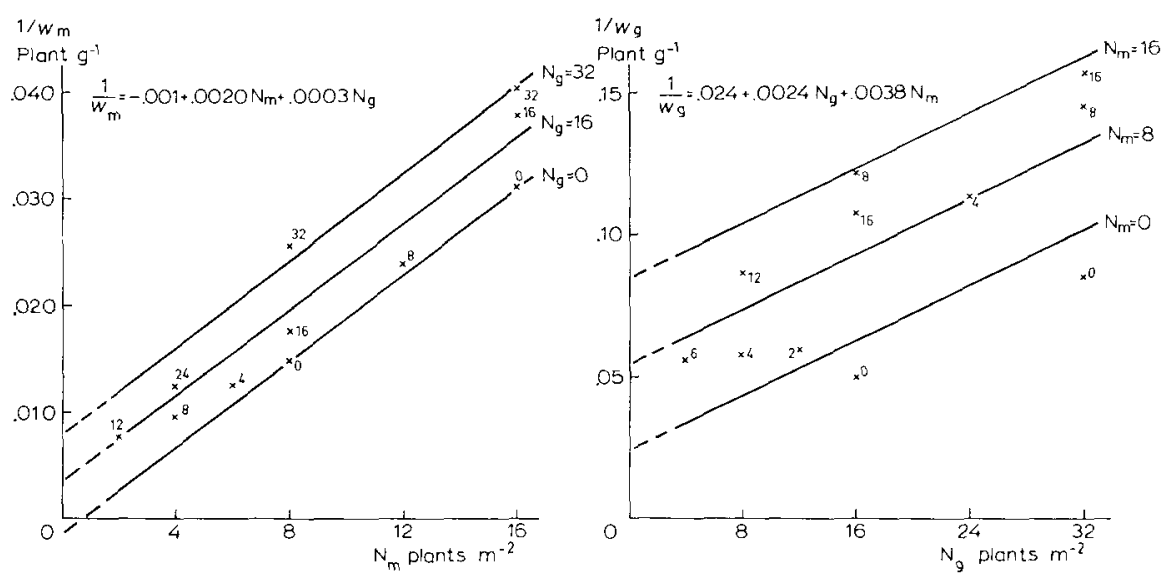

Fig. 3. Effect of number of maize plants $\left(N_{m}\right)$ and number of groundnut plants $\left(N_{g}\right)$ on the reciprocal of per-plant biomass of maize and on that of groundnut. In the plot with $1 / w_{m}$ subscripts of data points denote $N_{g}$ while in the plot with $1 / w_{g}$ subscripts denote $N_{m}$. Curves are those generated from the fitted multiple linear regression equations.

An analysis according to de Wit requires the use of replacement series (Spitters, 1980). A replacement series is the result of generating a range of populations by starting with a monoculture of one species and progressively replacing plants of that species with plants of the other species until a monoculture of the latter is produced. In contrast to de Wit's model, the present approach does not have the prerequisite of replacement series.

Mixed cropping experiments are intended to find mixtures that yield better than the monocrops, although there are several other reasons why mixed cropping is practised. However, when a mixture results in a yield benefit, the resources are exploited more efficiently in that mixture than in the monocrops. This suggests that the optimal density is higher for the mixture than for either of the monocrops. This is supported by experimental evidence (Willey, 1979, p. 74). For a realistic evaluation of mixtures an ascending progression of total densities is therefore required: an addition series. In comparison with replacement series, such addition series have the advantage of a better correspondence with the practice of mixed cropping. However, the disadvantage is that until now no model existed to analyse the competition effects in such a diverse series of populations. The presented model fills up this gap.

\section{Estimating competition effects from a diverse set of populations: An example}

The presented analysis will be illustrated with a set of data of maize and groundnut grown in mixtures and monocultures at different total densities.

\section{Descriptive equations}

The biomass data of maize are fitted by the multiple linear regression equation 


$$
1 / w_{m}=-0.001+0.0020 N_{m}+0.0003 N_{g}
$$

where $w_{m}$ is the average weight in grams per plant of maize, $N_{m}$ the number of maize plants and $N_{g}$ the number of groundnut plants per $\mathrm{m}^{2}$.

The biomass data of groundnut are described by

$$
1 / w_{g}=0.024+0.0024 N_{g}+0.0038 N_{m}
$$

We may present this graphically by plotting the reciprocal of the per-plant weight of a species against the number of plants of that species (Fig. 3). Parallel isolines connect populations with an equal number of plants of the other species.

We note that the data of maize are fitted very well by the model $\left(R^{2}=0.99\right)$. For the groundnut data the degree of fit is less, although yet $86 \%$ of the variation in biomass is accounted for by the regression equation $\left(R^{2}=0.86\right)$. Especially the reciprocal per-plant weights of groundnut in monoculture are overestimated. That is, the monocrop biomass of groundnut per unit area tends to be underestimated.

The advantage of presenting the data as in Fig. 3 is that deviations from the model are easily identified. In inspecting the data points, one should take into account the heterogeneity of variances that causes a greater error variation around $1 / w$ at larger values of $1 / w$ (Appendix 1).

The negative estimate of $b_{0}$ for maize is probably caused by random errors as its value is not significantly smaller than zero $\left(b_{0}=-0.0014 \pm 0.0010\right)$. However, the consequence of the negative value is that the model predicts a per-unit area biomass for the maize monocrop that increases when the stand gets more sparse. Of course, this is nonsense. It shows that extrapolation outside the data range should oe done with great caution. The equations describe the effects only within the data range studied.

\section{Competitive ability}

From the multiple linear regression equations we derive for the per-plant biomass of maize

$$
w_{m}=1 /\left(-0.001+0.0020 N_{m}+0.0003 N_{g}\right)
$$

and for that of groundnut

$$
w_{g}=1 /\left(0.024+0.0024 N_{g}+0.0038 N_{m}\right)
$$

We see that one maize plant and 6.9 groundnut plants have an equal influence on the average weight per plant of maize $(6.9=0.002040 / 0.000296$ being the extended form of $0.0020 / 0.0003$ of the equation of $w_{m}$ ). Hence, the addition of any extra maize plants to the mixture reduces the average weight of maize plants to the same extent as the addition of 6.9 groundnut plants. We may say that maize senses the presence of one maize plant as strongly as the presence of 6.9 groundnut plants. From the point of maize, maize itself is a 6.9 times stronger competitor than groundnut. 
Thus, in general terms: from the point of species 1 , the relative competitive ability of species 1 compared to 2 is defined by the ratio of the regression coefficients $\left.b_{1,1} / b_{1,2}\right)$. The coefficient $b_{0}$ is not involved in this expression as it characterizes the per-plant weight in an environment without interplant competition. The reciprocal $1 / b_{0}$ is the virtual weight of an isolated plant.

\section{Niche differentiation}

From the descriptive equation for the per-plant biomass of groundnut we derive that for groundnut the presence of one maize plant is similar to the presence of 1.6 $(=0.0038 / 0.0024)$ other groundnut plants. For maize this ratio was 6.9 . The influence of a maize plant, expressed relative to the influence of a groundnut plant, is therefore greater for maize itself than for groundnut. Intra-specific competition is greater than inter-specific competition. So, the species are only partly limited by the same resource; they partly avoid each other. This is defined as niche differentiation.

In mathematical terms: there is niche differentiation when $b_{1,1} / b_{1,2}>b_{2,1} / b_{2,2}$ or, in other words, when the double quotient $\left(b_{1,1} / b_{1,2}\right) /\left(b_{2,1} / b_{2,2}\right)$ exceeds unity. For the maize/groundnut experiment we find a value of $6.9 / 1.6=4.3$. This double quotient may be written as the product $\left(b_{1,1} / b_{1,2}\right) \times\left(b_{2,2} / b_{2,1}\right)$ and measures the degree of niche differentiation.

In mixtures of grain crops, such as maize, with legumes, such as groundnut, niche differentiation is the rule. The mechanisms of niche differentiation were reviewed and discussed by Trenbath (1976), Spitters (1980) and Braakhekke (1980).

Usually, the degree of niche differentiation is measured with the relative yield total (de Wit, 1960; de Wit \& van den Bergh, 1965). The relative yield total is the sum of the relative yields, with the relative yield of a species being the yield of that species relative to its yield in monoculture. In formula, the relative yield total

$$
\mathrm{RYT}=\mathrm{RY}_{1}+\mathrm{RY} Y_{2}=y_{12} / y_{11}+y_{21} / y_{22}
$$

with the yields $y$ in grams per unit area and the subscripts denoting the species. It is emphasized that the monoculture yields are those from the replacement series studied and not the yields of any other monoculture. With that, RYT is associated invariably with the replacement principle. In the maize/groundnut experiment, RYT was greater than one. This agrees with the earlier conclusion of niche differentiation which was based on the product $\left(b_{1,1} / b_{1,2}\right) \times\left(b_{2,2} / b_{2,1}\right)$.

In demonstrating niche differentiation, this product has, compared with RYT, the advantages that (a) it can be estimated directly from a wide range of populations differing in total density without requiring replacement series and that (b) its value is independent of the plant number of each species.

\section{Marketable yield}

The competition effects were analysed on the basis of biomass. In a subsequent paper (Spitters, 1983) it will be discussed how the findings for biomass have to be transposed to marketable yield and how the effects of mixed cropping can be interpreted. 


\section{References}

Braakhekke, W. G., 1980. On coexistence: a causal approach to diversity and stability in grassland vegetation. Agric. Res. Rep. (Versl. landbouwk. Onderz.) 902: 164 pp.

Kempthorne, O. \& L. Folks, 1971. Probability, statistics, and data analysis. Iowa State University Press, Ames, Iowa, $555 \mathrm{pp}$.

Nelder, J. A., 1962. New kinds of systematic designs for spacing experiments. Biometrics 18: 283-307.

Papendick, R. I., P. A. Sanchez \& G. B. Triplett (Eds), 1976. Multiple cropping. American Society of Agronomy, Special Publication, 27: $378 \mathrm{pp}$.

Spitters, C. J. T., 1979. Competition and its consequences for selection in barley breeding. Agric. Res. Rep. (Versl. landbouwk. Onderz.) 893: 268 pp.

Spitters, C. J. T., 1980. Competition effects within mixed stands. In: R. G. Hurd, P. V. Biscoe \& C. Dennis (Eds), Opportunities for increasing crop yields. Pitman, London, pp. 219-231.

Spitters, C. J. T., 1983. An alternative approach to the analysis of mixed cropping experiments. 2. Marketable yield. Neth. J. agric. Sci. 31 (in press).

Steel, R. G. D. \& J. H. Torrie, 1960. Principles and procedures of statistics. McGraw-Hill, New York, $481 \mathrm{pp}$.

Trenbath, B. R., 1976. Plant interactions in mixed crop communities. In: R. I. Papendick, P. A. Sanchez \& G. B. Triplett (Eds), Multiple cropping. American Society of Agronomy, Special Publication 27: 129-169.

Trenbath, B. R., 1978. Models and the interpretation of mixture experiments. In: J. R. Wilson (Ed.), Plant relations in pastures. CSIRO, Melbourne, pp. 145-162.

Willey, R. W., 1979. Intercropping-its importance and research needs. Field Crop Abstr. 32: 1-10, 7385.

Willey, R. W. \& S. B. Heath, 1969. The quantitative relationships between plant population and crop yield. Adv. Agron. 21: 281-321.

Wit, C. T. de, 1960. On competition. Agric. Res. Rep. (Versl. landbouwk. Onderz.) 66(8): $82 \mathrm{pp}$

Wit, C. T. de \& J. P. van den Bergh, 1965. Competition between herbage plants. Neth. J. agric. Sci. 13: $212-221$.

\section{Appendix 1. Weighted regression and statistical analysis}

We may derive an approximate variance for the reciprocal of the weight per plant $w$ by the method of statistical differentials (e.g. Kempthorne \& Folks, 1971, p. 130). This gives

$$
\operatorname{var} 1 / w \simeq(1 / \varepsilon w)^{2} \operatorname{var} w
$$

where $\varepsilon w$ is the expected value of the average per-plant weight $w$ of the species in the population considered.

Per-plant weights may vary considerably among populations. It seems therefore more appropriate to assume a constant variation coefficient than to assume var $w$ to be constant over populations (Spitters, 1979, p. 89). That gives

$$
\operatorname{var} w_{i} /\left(\varepsilon w_{i}\right)^{2}=\operatorname{var} w_{j} /\left(\varepsilon w_{j}\right)^{2} \text { or } \operatorname{var} w_{i}=\left(\varepsilon w_{i} / \varepsilon w_{j}\right)^{2} \operatorname{var} w_{j}
$$

where $i$ and $j$ denote to two different populations. Combining this expression with that for $\operatorname{var}(1 / w)$ results into

$$
\operatorname{var} 1 / w_{i} \simeq\left\{\left(1 / \varepsilon w_{i}\right) /\left(1 / \varepsilon w_{j}\right)\right\}^{2} \operatorname{var} 1 / w_{j}
$$


This shows that the error variation around $1 / w$ is considerably enhanced by an increase in $1 / w$ and therewith by an increase in population density. This should be kept in mind in interpreting the data points in plots like Fig. 3.

This hetereogeneity of errors makes it preferable to apply a weighted linear regression to estimate the competition coefficients. Usually, the reciprocals of the variances are used as weights. Hence, when the per-plant weight of a species in one population is 3 times that in another population, the first datum has to be weighted 9 times the latter in the regression analysis. For details see e.g. Steel \& Torrie $(1960$, pp. 180-181). The weighted regression analysis also provides the standard errors of the competition coefficients.

The weighting to the reciprocal of the variance assumes that the effect of differences in sample size among the populations on the per-plot variance is negligible, which requires that the number of plants of eacht species per plot is not too small.

\section{Appendix 2. Comparison with de Wit's model}

\section{Density response}

De Wit (1960, p. 33) also assumed a hyperbolic relation between biomass and number of plants per unit area:

$$
y=\{\beta N /(\beta N+1)\} \Omega
$$

with $\beta$ and $\Omega$ being the constants characterizing the hyperbola. Comparing the linear relation for the reciprocal of the weight per plant

$$
1 / w=N / y=1 / \beta \Omega+(1 / \Omega) N
$$

with our linear regression equation, we find $b_{0}=1 / \beta \Omega$ and $b_{1}=1 / \Omega$. De Wit $(1960$, p. 33) gives a physiological interpretation of $\beta$ and $\Omega$.

\section{Mixture effect}

De Wit (1960, p. 60) extended this density function to allow for competition between different species in dependence on plant density. For the biomasses of two species 1 and 2 grown together in a mixture, this extension results into

$$
y_{1}=\left\{\beta_{1} N_{1} /\left(\beta_{1} N_{1}+\beta_{2} N_{2}+1\right)\right\} \Omega_{1}
$$

and

$$
y_{2}=\left\{\beta_{2} N_{2} /\left(\beta_{1} N_{1}+\beta_{2} N_{2}+1\right)\right\} \Omega_{2}
$$

The same $\beta$ s occur in both equations so that the equations are mutually dependent. From this interdependence, it can be derived that the expressions hold only when RYT $=1$, i.e. when there is no niche differentiation. However, niche differentiation is a prerequisite for yield advantage of mixed cropping, so that mixed cropping 
studies deal generally with situations where RYT $>1$. The model of de Wit (1960) is therefore not applicable to estimate competition effects from a set of populations varying in total density. To estimate competition effects and the degree of niche differentiation with the model of de Wit, replacement experiments are necessary.

In contrast to the model of de Wit, the presented approach enables to estimate competition effects from populations differing in total density because it uses independent equations for the different species. It enables also a direct estimate of the degree of niche differentiation in such a data set by using another measure of niche differentiation. Moreover, a statistical analysis of the competition effects is given (Appendix 1).

From the multiple linear regression equation for the reciprocal of the biomass per plant, it can be seen that $b_{1,0}=1 / \beta_{1} \Omega_{1} ; b_{1,1}=1 / \Omega_{1} ; b_{1,2}=\beta_{2} / \beta_{1} \Omega_{1} ;$ and $b_{1,1} / b_{1,2}=$ $\beta_{1} / \beta_{2}$.

\section{Replacement series, competitive ability}

In the analysis of de Wit, replacement experiments are essential in the estimation of competition effects and in demonstrating niche differentiation. According to de Wit $(1960$, p. 15), the biomass of species 1 in a replacement series with species 2 is given by

$$
y_{1,2}=\left\{k_{12} N_{1} /\left(k_{12} N_{1}+N_{2}\right)\right\} y_{1,1}
$$

where $y_{1,1}$ is the biomass of species 1 in monoculture at a density of $N_{1}+N_{2}$. The 'relative crowding coefficient' $k_{12}$ measures the competitive ability of species 1 with respect to species 2 . It can be derived that

$$
\begin{aligned}
& k_{12}=\left\{1+\beta_{1}\left(N_{1}+N_{2}\right)\right\} /\left\{1+\beta_{2}\left(N_{1}+N_{2}\right)\right\}=\left\{b_{1,0}+b_{1,1}\left(N_{1}+N_{2}\right)\right\} /\left\{b_{1,0}+\right. \\
& \left.+b_{1,2}\left(N_{1}+N_{2}\right)\right\}
\end{aligned}
$$

Thus $k_{12}$ is considered to be independent of the relative frequencies of the species in the mixture and only to be dependent on the total density $N_{1}+N_{2}$ of the replacement series.

In the approach presented in this paper, the competitive ability of species 1 to species 2 is measured by $b_{1.1} / b_{1,2}$. This ratio denotes that a plant of species 1 feels the presence of one other plant of that species like the presence of $b_{1,1} / b_{1,2}$ plants of species 2 . Compared with the relative crowding coefficient $k_{12}$, this ratio has the advantages of being easier to interpret and being independent of total population density. 